\title{
LA INFLUENCIA DE OBJETIVOS COMUNICATIVOS EN LA DIDACTIZACION DE CONTENIDOS GRAMATICALES
}

\author{
Maria A. Borrueco Rosa
}

\section{INTRODUCCIÓN}

Punto de partida de la presente reflexión sobre didáctica y metodología en torno al tiempo verbal en la enseñanza de $\mathrm{DaF}$ es la preocupación de todo docente por mejorar la producción lingüística de sus alumnos. En este sentido son numerosos y muy diferentes los intentos por formular una teoría clara acerca del proceso de adquisición de lenguas extranjeras $^{1}$. Aún siendo conscientes de que el proceso de aprendizaje es un proceso individual, determinado por diferentes factores ${ }^{2}$ (neurofisiológicos, cognitivos, socioeconómicos, socioculturales, afectivos, etc.) consideramos que el discente que no está inmerso en el entorno natural de la lengua objeto de estudio, no está en condiciones óptimas de formular reglas sobre el sistema lingüístico por sí mismo ${ }^{3}$. Es por ello que la labor desempeñada por el docente resulta decisiva en el mencionado proceso de adquisición de lenguas extranjeras, pues éste se produce bajo la influencia de determinados mecanismos organizados con un fin específico. La intencionalidad del docente será la que origine los objetivos tanto didácticos como metodológicos y éstos, en última instancia, los resultados. Todas las estrategias metodológicas, la didactización de contenidos de diferente naturaleza por parte del docente y la labor propia del estudiante tienen un único y último fin: que éste llegue a ser comunicativamente competente. Se trata, pues, de potenciar la capacidad comunicativa activa-productiva y receptiva-pasiva del estudiante.

Toda situación comunicativa supone un nivel de relación social entre los participantes. Elementos constitutivos del acto comunicativo son, entre otros, los datos referentes al lugar,

${ }^{1}$ Vid. a este respecto Arbeitsbruppe Fremdsprachenerwerb Bielefeld (1995): «Fremdsprachenerwerbsspezifische Forschung. Aber wie? Theoretische und methodologische Überlegungen (I)». En: Deutsch als Fremdsprache, 3, Leipzig, 1996, pp. 144-155.

2 Vid. A. Bogner, «Fremdsprachenerwerbsforschung zwischen Kultur- und Naturwissenschaft». En: Jahrbuch Deutsch als Fremdsprache, 25, 1999, pp. 29-49

${ }^{3}$ B. Mulo, «Aspekte einer kommunikativ-funktionalen Grammatik aus der Fremdperspektive». En: Deutsch als Fremdsprache", 3, 1996, pp. 156-159. 
tiempo y tema ${ }^{4}$. En este marco se manifiestan los contenidos relativos al paradigma verbal como muy importantes, tanto por la frecuencia con la que el tiempo verbal ha de ser producido como por la función que desempeña en el conjunto. Los parámetros temporales han de quedar determinados con claridad para que la comunicación ${ }^{5}$, el intercambio de información, sea efectiva. Pero la enseñanza y aprendizaje (didáctica y metodología) de contenidos gramaticales, entre los que situamos toda la información relativa al tiempo verbal, resulta tan importante como conflictiva y difícil de abordar. Se trata de una tarea ardua y árida para estudiantes y docentes, tanto más si la enseñanza de la gramática se evidencia en numerosas ocasiones como ineficaz y contraproducente ${ }^{6}$. A pesar de esta evidencia sabemos que resulta imprescindible para actos de habla y escritura un sistema de reglas y normas, estructurado jerárquicamente y fundamentado en la lengua estándar?. Pero la imposibilidad de renunciar a la descripción del sistema lingüístico produce como consecuencia un alto grado de desinterés en el estudiante, más motivado por una enseñanza funcional y situativa. Surge así el conflicto: somos conscientes, por un lado, de la necesidad de una gramática didáctica, centrada en el proceso de aprendizaje, en el discente y en sus necesidades comunicativas pero, por otro, resulta muy difícil integrar metodológicamente estos contenidos en una enseñanza que persiga como objetivo máximo la comunicación.

\section{GRAMÁTICA Y COMUNICACIÓN}

El docente ha de didactizar de una forma u otra unos contenidos gramaticales. De hecho, desde que en la literatura científica irrumpe la preocupación por la adquisición de lenguas extranjeras son numerosas y diversas las aproximaciones a la enseñanza de la gramática ${ }^{8}$. Los métodos basados en la traducción que no conducían al desarrollo de destrezas comunicativas o los métodos basados en el pattern que supusieron una excesiva valoración de la automatización, hacen que surja con fuerza la orientación comunicativa en la enseñanza. Orientación comunicativa que aparece asociada a otros aspectos, como los psicológicos, además de los puramente estructurales o formales. Pero la euforia "comunicativa" tiene como consecuencia un desvío absoluto de la descripción sistemática

4 K. Deutrich / G. Schank, «Situationsspezifischer Einsatz sprachlicher Mittel. Anregungen für den Deutschunterricht». En: Der Deutschunterricht, 6, 1973, 100-120.

${ }^{5}$ Irmhild Wragge aporta la siguiente definición: «Unter Kommunikation verstehen wir das Inbeziehungstreten von zwei oder mehreren menschlichen Individuen oder Gruppen zum Zwecke des Informationsaustausches.» Vid. I. Wragge, «Kommunikationsfähigkeit als Aufgabe des Deutschunterrichts. Ein Beitrag zur Didaktik des Sprachtrainings». En: Der Deutschunterricht, 3, 1974, pp. 82-98.

${ }^{6}$ Vid. a este respecto E. Diehl, «Vom Deutschunterricht zum Deutscherwerb? Das Genfer DiGS-Projekt». En: Deutsch als Fremdsprache, 3, 1998, pp. 162-168 o M., Thurmair, «Nicht ohne meine Grammatik!». En: Jahrbuch Deutsch als Fremdsprache, 23, 1997, pp. 25-45

${ }^{7}$ Vid., por ejemplo, G. Wolff, «Sprechakte im sozialen Kontext». En: Der Deutschunterricht, 6, 1973, pp. 19-49 o L. Götze, «Grammatikmodelle und ihre Didaktisierung in Deutsch als Fremdsprache». En: Deutsch als Fremdsprache, 3, 1996, pp. 136-143.

8 Gramática entendida en el sentido de D. Ader, «Grammatik als Sprachförderung». En: Handbuch Deutschunterricht, Bd. 1, Düsseldorf, Schwann, 1983, pp. 399-410. 
de la lengua, de normas del sistema lingüístico que al ser transgredidas dificultan seriamente la comunicación verbal. ${ }^{9}$

La convicción de que forma y función ${ }^{10}$ deben ir unidas conduce a un nuevo concepto de enseñanza, que de alguna forma integre ambos aspectos: se trata de centrar la atención en objetivos comunicativos pero sin dar la espalda a la gramática tradicional. Surge así la gramática comunicativa, no libre de controversia ${ }^{11}$. Autores como Helbig ${ }^{12}$ prefieren hablar de una gramática de orientación comunicativa, pues muy acertadamente defienden la idea de que la enseñanza no puede ser "comunicativa", pues durante el proceso de enseñanza/aprendizaje sólo podemos alcanzar a simular "situaciones" y sólo en escasas ocasiones se produce un proceso comunicativo real en el que el aprendiz de la lengua extranjera se muestra comunicativamente competente.

Esta gramática de orientación comunicativa no considera sólo la descripción del sistema lingüístico sino también la descripción del uso lingüístico. Se trata de una gramática al servicio de la comunicación, centrada en la función; una gramática en la que la descripción formal es un medio y no un fin en sí misma. El objetivo es transmitir reglas gramaticales desde situaciones comunicativas. La euforia comunicativa tendía a prescindir de una descripción sistemática de los contenidos gramaticales. Desde este enfoque se pretende didactizarlos atendiendo a objetivos comunicativos, objetivos centrados en la descripción del uso y determinados por el contexto y la situación. En todo caso se trata de una gramática didáctica, centrada en el propio proceso de aprendizaje, y que ha sufrido un proceso de adaptación con respecto a la gramática lingüística, pues predomina la función docente a la descriptiva.

Pero esta orientación comunicativa de la gramática no deja de suscitar numerosas dudas: ¿En qué medida resulta importante la confrontación del estudiante con las reglas y las normas del sistema lingüístico? ¿Cómo puede potenciar la gramática la competencia comunicativa? ¿Las reglas gramaticales no siguen siendo, al fin y al cabo, reglas gramaticales? ¿Aporta realmente algo positivo la gramática al proceso comunicativo? ¿El conocimiento sistemático de contenidos gramaticales es realmente necesario para que la comunicación verbal tenga lugar? Resultan de gran interés en este sentido las aportaciones de Irmhild Wragge ${ }^{13}$. Wragge establece claramente la diferencia entre competencia

${ }^{9}$ En el contexto de la definición aportada por Irmhild Wragge, op. cit. p. 85, entendemos que la transgresión de normas del sistema lingüístico dificulta el intercambio de información y, por lo tanto, la comunicación.

${ }^{10}$ Helbig previene de la práctica desmesurada de la competencia comunicativa en detrimento del sistema lingüístico y defiende una enseñanza que considere la unidad dialéctica entre forma y función. Vid, G. Helbig, «Kommunikativer Grammatikunterricht - Ziele, Möglichkeiten und Grenzen -». En: Deutsch als Fremdsprache, 1, Leipzig, 1986, pp. 14-20.

${ }^{11}$ Autores como Admoni no admiten ni justifican la necesidad de "nuevas" gramáticas, pues en su opinión los aspectos comunicativos tienen proyección en la gramática tradicional. Citado por G. Helbig, op. cit. pág. 17.

${ }^{12}$ G. Helbig, op. cit., p. 18.

${ }^{13}$ I. Wragge, op. cit., pp. 91-93 
lingüística y competencia comunicativa. La competencia lingüística, que supone el conocimiento amplio de reglas y normas gramaticales, está inmersa en un proceso más amplio: el de la competencia comunicativa. El modelo propuesto por Wragge establece la siguiente relación:

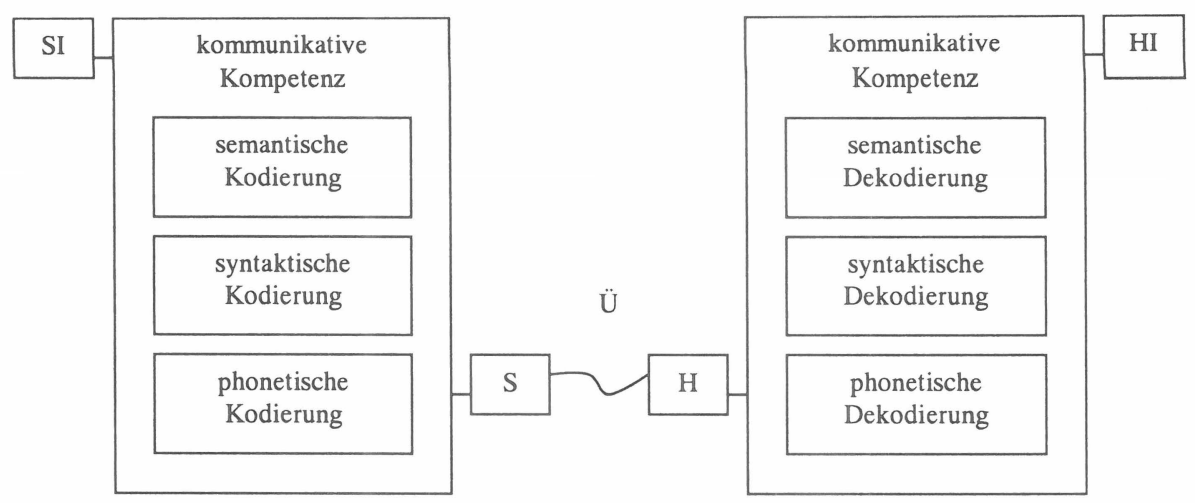

SI=Sprecherintention S=Sprecher Ü=Übertragung $\mathrm{H}=$ Hörer $\mathrm{HI}=$ Hörerinformation

Si los objetivos programados por el docente se limitan al aspecto formal, el discente será capaz de generar oraciones gramaticalmente correctas, contribuyendo así a su competencia lingüística, pero aportaremos muy poco al desarrollo de su capacidad comunicativa, pues se trata de productos abstractos, sin relación contextual. El aprendizaje de lenguas extranjeras requiere necesariamente la práctica comunicativa.

Pero de igual forma la competencia comunicativa supone además de la codificacion y decodificación pragmática, situacional, la codificación y decodificación de información gramatical. Ambos aspectos forman una unidad inseparable, unidad que se debe manifestar también a nivel metodológico. Por ello, durante el proceso de confrontación con contenidos gramaticales debemos partir de situaciones que simulen el intercambio de información, en definitiva, la comunicación. Si resulta importante programar objetivos que vayan más allá de la competencia lingüística, es decir, contextualizar contenidos gramaticales en situaciones que simulen la comunicación para potenciar las destrezas comunicativas, de la misma forma debemos considerar que la competencia comunicativa presupone la competencia lingüística. Es decir, la comunicación verbal no es posible si no estamos en posesión de determinados conocimientos gramaticales. La capacidad comunicativa es potenciada cuando consideramos en nuestros objetivos docentes componentes de la competencia lingüística (codificación/decodificación semántica, sintáctica y fonética) y de la competencia comunicativa (codificación/decodificación pragmática) de forma 
interrelacionada. En este sentido consideramos la aportación de la gramática a la comunicación como muy importante.

Las críticas que ha sufrido la enseñanza y el aprendizaje de la gramática por parte de discentes y también docentes tienen su origen, sin duda, en el hecho de no contextualizar los contenidos gramaticales e incluirlos en un marco más amplio, el de la competencia comunicativa. Es por ello de suma importancia considerar la interrelación gramática y comunicación como esencial antes de abordar cualquier reflexión metodológica.

\section{CONSIDERACIONES METODOLÓGICAS EN TORNO AL TIEMIPO VERBAL。}

Si centramos nuestra reflexión en el tiempo verbal desde la perspectiva de una gramática de orientación comunicativa, habremos de considerar factores que generalmente no son tenidos en cuenta. Factores que potencien aspectos funcionales del proceso de adquisición de lenguas. Desde un punto de vista metodológico habremos de invertir el proceso de enseñanza. No partiremos de la forma para llegar a la función, sino de la intención comunicativa para llegar a la forma o a todas las formas posibles que nos brinda el sistema ${ }^{14}$. Nuestro objetivo último, el desarrollo de la capacidad comunicativa, se fundamenta en un objetivo más genérico y amplio, a saber, el de potenciar una enseñanza emancipatoria aunque durante el proceso de enseñanza y aprendizaje no podamos eludir estrategias compensatorias.

Partimos de la idea de que la confrontación del discente con los contenidos influye absolutamente en la percepción, comprensión y asimilación de los mismos. Consideramos, en relación con lo expuesto hasta este momento, tres estrategias como válidas en nuestro intento de relacionar contenidos gramaticales, en nuestro caso el tiempo verbal, y proceso comunicativo: la presentación del INPUT de forma contextualizada, a través de paradigmas y a través de la reflexión interactiva ${ }^{15}$.

\subsection{Contextualización del INPUT}

Como ya hemos mencionado con anterioridad, consideramos inadecuado confrontar al estudiante con rígidas reglas sin presentar de forma simultánea la intención comunicativa que fundamenta el contenido gramatical. La función, enmarcada en un contexto, será nuestro punto de partida. Es decir, la didactización del INPUT gramatical debe estar basado en modelos de comunicación. La necesidad comunicativa del discente debe fundamentar y dar origen a la búsqueda de elementos lingüísticos en el sistema.

Veamos en este sentido el ejemplo ofrecido por Rusch ${ }^{16}$ para ilustrar este tipo de metodología. Cita el autor el capítulo 18 titulado "Leben im Alter" perteneciente al libro de

${ }^{14}$ Vid. B. Mulo, op. cit., p. 156.

15 Dejamos a un lado factores lingüísticos y extralingüísticos que influyen en el proceso de enseñanza y aprendizaje no pertinentes aquí como el perfil previo de los estudiantes, progresos, regresiones o fosilizaciones individuales.

${ }^{16}$ P. Rusch, «Schritte zum Ausbau einer Lernergrammatik». En: Deutsch als Fremdsprache, 4, Leipzig, 1998, pp. 233-238. 
texto "Moment mal. Bd. 2". El discente es confrontado en principio con una doble página que reproduce parte del album de fotos de uno de los personajes del capítulo. Esta circunstancia determina la dimensión "tiempo" que será abordada a lo largo del capítulo. A través de textos escritos y hablados se va desplegando la situación que abarcará todo el sistema temporal: el recuerdo que evocan las fotos, tanto a nivel personal como social, pues se introduce la referencia a unos datos estadísticos de circunstancias propias de ese momento pasado. Los estudiantes ya han entrado en contacto con los tiempos verbales presente (Präsens) y pretérito perfecto (Perfekt). El contenido nuevo, en este caso, el pretérito imperfecto (Präteritum) es presentado de forma contextualizada. No se fundamenta en la forma sino en la situación: el tiempo verbal presente (Präsens) constituye el marco de los datos autobiográficos del personaje reflejados en el uso del perfecto (Perfekt) y los datos estadísticos asociados al lenguaje escrito quedan expresados a través del imperfecto (Präteritum). El docente habrá de conducir al estudiante hasta que éste pueda interpretar el contexto y determinar la diferenciación temporal. El contexto nos conduce así a la forma y da inicio al proceso de adquisición de nuevos contenidos.

Este tipo de metodología, situacional, que posibilita la inmersión en información contextualizada favorece además la motivación del estudiante y su predisposición al aprendizaje de áridas reglas gramaticales. Pero las estrategias docentes no deben limitarse a la presentación e inmersión contextual y, en todo caso, a la prescripción de normas. El estudiante necesita un acercamiento diferente al sistema, que evite la memorización de infinitas tablas y le faciliten el paso a la aplicación intuitiva de sus conocimientos. La inmersión contextual es tan sólo una parte del proceso de enseñanza, que no da solución por sí misma a la gran cantidad de dificultades a las que se enfrenta el estudiante de un sistema lingüístico en ocasiones muy diferente al materno. La consecución de objetivos comunicativos requiere a veces la aplicación de estrategias que en sí mismas no son comunicativas, pero que conducen al desarrollo de destrezas que sí lo son.

\subsection{Presentación paradigmática}

El estudiante de lenguas extranjeras suele tener contacto con las estructuras de la nueva lengua generalmente de forma sintagmática. Estas estructuras quedan almacenadas y van conformando su acervo lingüístico. El paso de este estado a un conocimiento paradigmático de la lengua supone un proceso muy relevante en el complicado proceso de adquisición de lenguas extranjeras ${ }^{17}$. Toda necesidad comunicativa corresponde a un ámbito de expresión que se materializa en un paradigma lingüístico. Así, antes de confrontar al discente con aspectos teóricos o prácticos sobre el tiempo verbal es recomendable presentarle, por ejemplo, todas las posibilidades que encontramos en la lengua para referirnos a la dimensión temporal ${ }^{18}$ :

1. Tempus-Formen des Verbs

2. Adverbien oder
Er ging ...

Gestern...

\footnotetext{
${ }^{17}$ Vid. a este respecto P. Rusch, op. cit., p. 234 o B. Mulo, op. cit., p. 157.

${ }^{18}$ En este mismo sentido vid. L. Götze, op. cit., p. 141.
} 
adverbiale Ausdrücke

3. Adjektive oder PI / PI
Eines Morgens...

Das letzte Jahr

Die kommende Woche...

El INPUT inicial correspondiente, por ejemplo, a la intención comunicativa "futuro" no se limitaría al ejemplo (1) y menos aún a una presentación descontextualizada de formas gramaticales (2):

(1) Ich werde das Haus kaufen.

(2) ich werde

du wirst

er, sie, es wird

wir werden

$+\quad$ infintivo

ihr werdet

sie werden

Por el contrario, se hace necesaria una presentación escalonada (atendiendo a la progresión natural del discente) del INPUT, en forma de paradigmas sucesivos y, como hemos mencionado en el punto anterior, contextualizados. Citamos a modo de ejemplo algunos:

(a) Paradigma de la dimensión "tiempo" y su correspondencia con los tiempos verbales gramaticales:

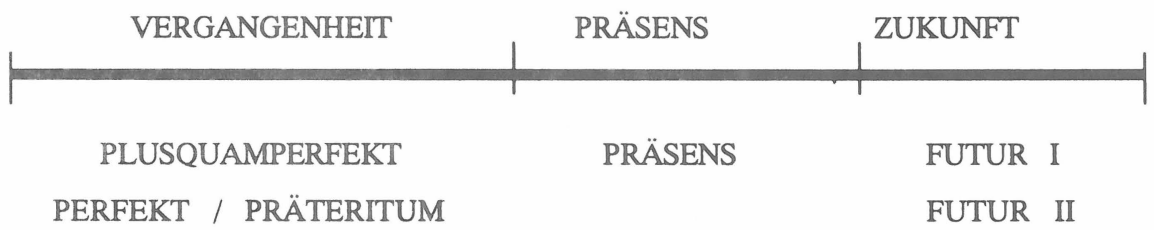

Para llegar a la forma temporal futuro partiremos de un contexto, y desde el contexto de un paradigma (a). Inmerso en este paradigma el discente debe llegar a ser consciente de la diferencia existente entre la dimensión "tiempo", concepto universal, y la existencia de diferentes tiempos gramaticales. El esquema estructural paradigmático le ayudará posteriormente a situar sus intenciones comunicativas relacionadas con los parámetros temporales.

Esta primera aproximación no le permitirá aún asimilar que la correspondencia del sistema temporal y los tiempos gramaticales no es siempre objetiva y que, por el contrario, la relación tiempo universal y tiempo gramatical está en numerosos casos sujeta a una mayor complejidad, determinada por la inclusión en el discurso de elementos léxicos que 
también pueden expresar y situar la acción verbal en el pasado, presente o futuro, estableciendo una compleja correspondencia no objetiva y lógica entre temporalidad y formas gramaticales temporales. A esto habremos de añadir el uso absoluto y relativo de los tiempos, en el que se mezclan criterios no sólo sujetos al acto de habla o la perspectiva del hablante, sino también la consideración de factores como la anterioridad, simultaneidad o posterioridad con respecto al acto de habla. Esta interpretación, muy compleja para el discente puede ser ilustrada ampliando de forma progresiva su visión paradigmática.

(b) Paradigma de mecanismos lingüísticos posibles para una misma intención comunicativa:

Una vez establecido el contexto, diferenciada la dimensión "tiempo" (Vergangenheit, Präsens, Zukunft) y establecida la correspondencia objetiva con los tiempos gramaticales podremos ofrecer al estudiante el paradigma comentado en (a) ampliado:

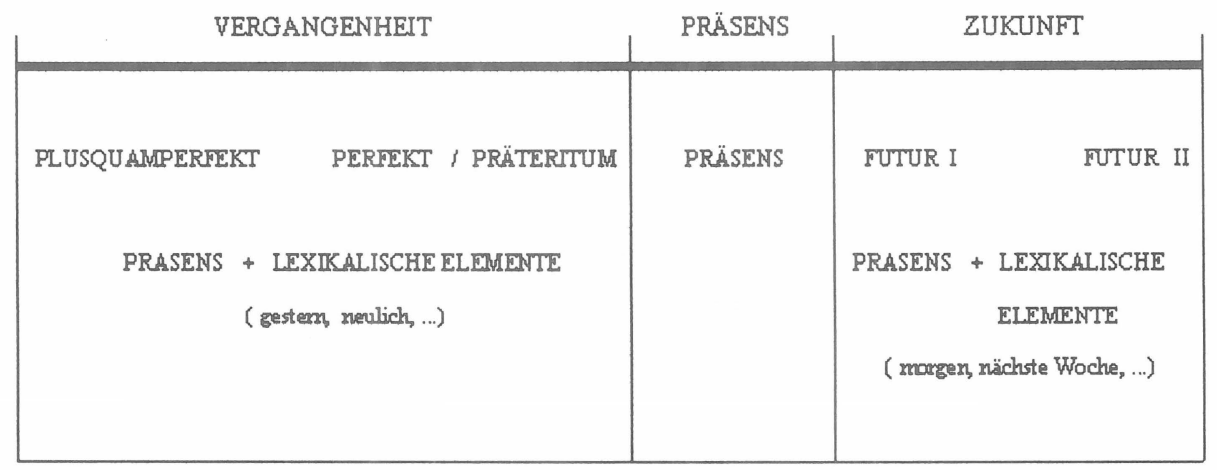

Se contempla aquí, por ejemplo, la existencia de dos variantes del presente gramatical (Präsens), a saber, la posibilidad de expresar acciones futuras (3) o pasadas (4):

(3) Wir treffen uns morgen.

(4) 1914 beginnt der erste Weltkrieg.

Como es lógico, la progresión lingüística del discente permite ampliar sus conocimientos hasta alcanzar un grado alto de complejidad. Por ejemplo el que ilustra el siguiente paradigma $^{19}$ :

19 No hemos considerado aquí otras variantes, como la descripción semántica referida a la capacidad de expresar modalidad, suposiciones o hipótesis como en Sie kommen vermutlich um $7.15 \mathrm{Uhr}$ an. Estas variantes tendrían que ser introducidas en fases posteriores, aunque insistimos, siempre de forma paradigmática. 


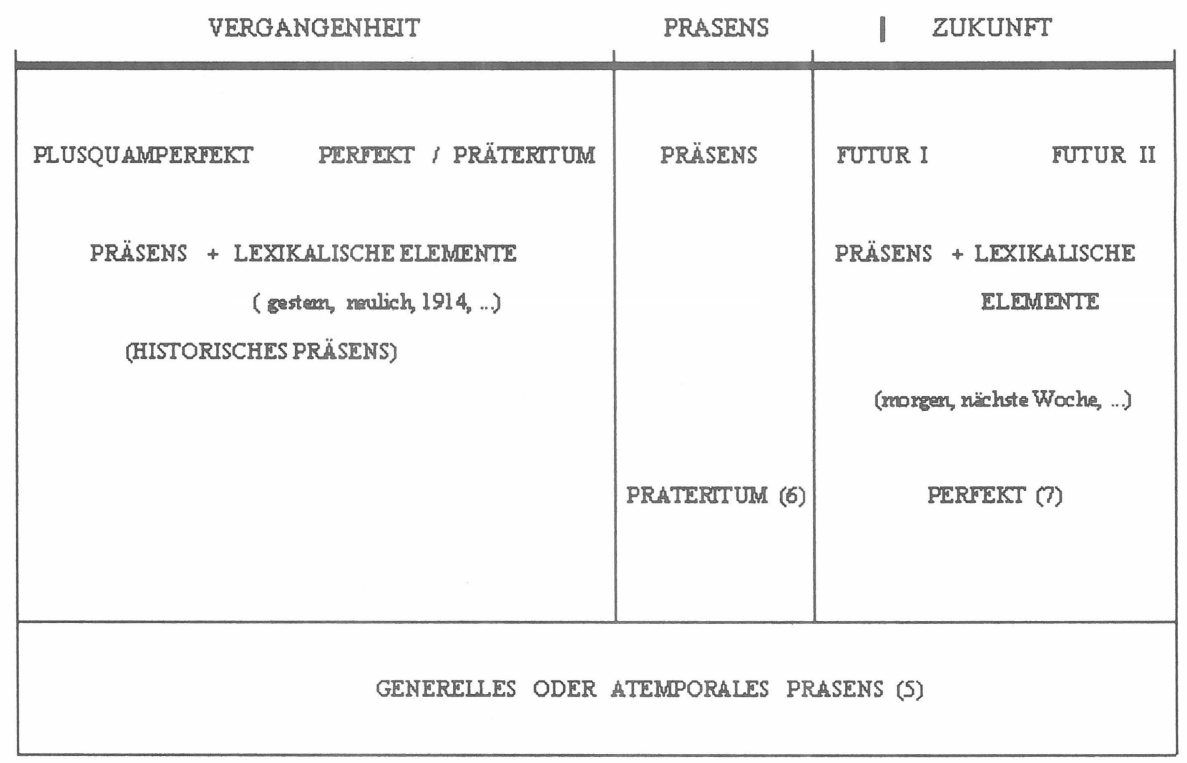

Queda confrontado el discente, en nuestro caso, con variantes como el presente histórico (4), el futuro (3) o el presente atemporal (5) expresados por el presente gramatical, por un lado, o con el pretérito imperfecto (6) en sustitución del presente, por otro, o con el pretérito perfecto expresando acciones futuras (7):

(5) Gold ist ein Edelmetall.

(6) Wir war doch der Name?

(7) Er hat sich das Buch bis Montag ausgeliehen.

El proceso comunicativo se caracteriza por su complejidad. Considerar los factores que influyen en la selección de una determinada opción resulta igualmente complejo. La progresiva competencia en el difícil proceso de adquisición de la lengua objeto de estudio es la que posibilita que el discente que tenga la necesidad comunicativa de expresar un hecho 0 acción futura, por ejemplo, actualice y active el paradigma correspondiente: 


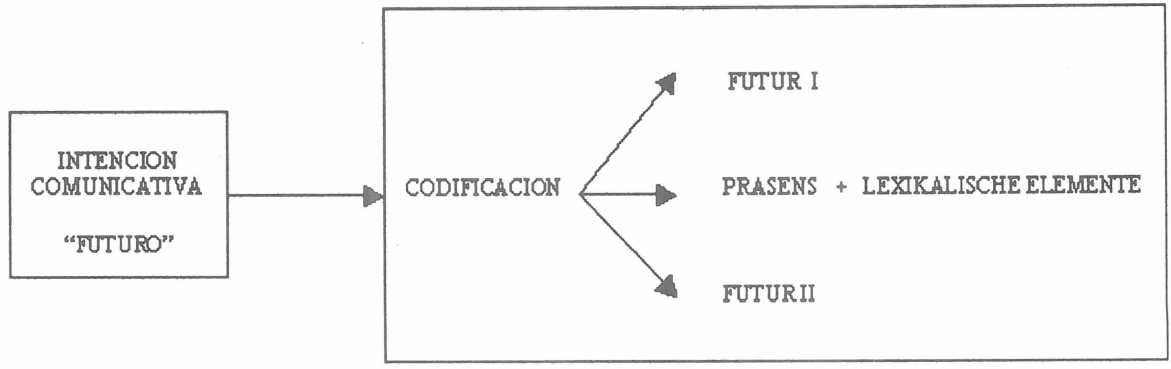

(c) Paradigma de las formas gramaticales:

Incluso en lo referente a la flexión verbal resulta adecuado presentar la información en forma de paradigma dando al discente la posibilidad de descartar las no pertinentes:

ich sage ich sagte ich habe gesagt ich werde sagen

0

$\begin{array}{llllll}\text { ich sage } & \text { ich sagte } & \text { ich habe gesagt } & \text { ich werde sagen } \\ \text { du sagst } & \text { du sagtest } & \text { du hast gesagt } & \text { du wirst sagen } \\ \text { er sagt } & \text { er sagte } & \text { er hat gesagt } & \text { er wird sagen }\end{array}$

Este tipo de confrontación con los contenidos no tiene como objetivo la memorización de infinitas tablas o paradigmas formales, por el contrario, pretende situar la estructura que vamos a analizar en profundidad. No es más que eso, una presentación del INPUT. En fases más avanzadas el discente podrá establecer divergencias y similitudes e interpretar aspectos formales con mayor seguridad, siempre desde la visión global del conjunto.

Los ejemplos descritos hasta el momento reflejan la intencionalidad del docente: confrontar al estudiante con contenidos gramaticales de tal forma que sea consciente de los paradigmas que necesitará cuando intente formular mensajes en la comunicación real. Se trata, por supuesto, de una fase en la que la activación de los contenidos gramaticales no es intuitiva, razón por la cual el aprendiz necesita de la aplicación de una metodología compensatoria. Aún así, estamos convencidos de que contribuye muy positivamente a la codificación sintáctica requerida en el acto comunicativo. La estrategia descrita se manifiesta así como válida para potenciar la unidad entre gramática y comunicación.

\subsection{Reflexión interactiva}

Si los objetivos docentes se centran básicamente en la instrumentalización del contenido gramatical al servicio de objetivos comunicativos, la integración de objetivos pragmáticos en la didactización de contenidos gramaticales nos lleva necesariamente a un diseño didáctico y metodológico diferente al tradicional, a objetivos metodológicamente emancipatorios además de compensatorios, comentados en los puntos anteriores. Destacan en este sentido estrategias docentes como la reflexión interactiva o, en nuestro caso 
concreto, la metacomunicación. Debemos insistir en que el proceso de aprendizaje no tiene por qué ser siempre comunicativo para conducir al desarrollo de la capacidad comunicativa.

Son muchas las ventajas de una presentación interactiva. En primer lugar nos ofrece la posibilidad de sensibilizar al discente y hacerle partícipe de los objetivos docentes en relación con el INPUT. Este acercamiento lleva necesariamente a una sensibilización lingüística que luego beneficiará su producción hablada y escrita, y sin duda la decodificación de información ${ }^{20}$. Por otro lado, estamos convencidos de que el aprendiz de la lengua extranjera, si es consciente de que instrumentalizamos los contenidos gramaticales, podrá desarrollar una capacidad de diagnóstico propia y localizar por sí mismo problemas, divergencias con la lengua materna o, cómo no, errores cometidos. Se trata, pues, de una tematización interactiva en la que los contenidos son desplegados de forma explícita y no implícita, de forma descriptiva y no prescriptiva.

La reflexión interactiva y el diagnóstico de dificultades presentes en el proceso de aprendizaje establecen la base para la resolución de problemas en futuras situaciones comunicativas reales. Esta es la auténtica contribución de la reflexión sobre gramática a la capacidad comunicativa del hablante, y es en este sentido en el que podemos hablar de una enseñanza emancipatoria ${ }^{21}$. Estrategias, a veces inductivas, otras deductivas son de gran ayuda para el discente, para al menos interpretar de forma segura las reglas en caso de no ser capaz de formularlas por sí mismo.

La metacomunicación nos permite abordar cuestiones desde la perspectiva del uso lingüístico, de tal manera que el estudiante llegue a ser consciente de los factores que pueden dificultar su futura competencia en un contexto real. Un aspecto que podemos tematizar, en relación con el paradigma verbal, junto a la descripción estructural, el uso coloquial de los tiempos verbales. Sirva de ejemplo, la progresiva tendencia a sustituir la información presente en las formas sintéticas por referencias contextuales o adverbiales ${ }^{22}$. El estudiante es confrontado, en esta ocasión, con el cambio sintáctico, Syntaktischer Wandel, representado por la aparición de parejas del tipo:

(8) Sie geht morgen ins Theater. / (9) Sie wird morgen ins Theater gehen.

La diversificación semántica presente en algunos tiempos gramaticales, comentada en el punto anterior como paradigma formal, se manifiesta al estudiante de nuevo pero a nivel de uso lingüístico. Así podrá saber que en textos hablados el hablante prefiere la forma

${ }^{20}$ Compárese en este sentido la tendencia, contraria a este acercamiento cognitivo al proceso de aprendizaje, desarrollada por la metodología puramente comunicativa, en la que los mecanismos lingüísticos se presentan de forma situacional, y se asimilan de forma reproductiva con intención de incorporarlos luego de forma creativa en el proceso comunicativo, renunciando a una enseñanza gramatical explícita. Vid. M. Thurmair, op. cit., p. 25.

${ }^{21}$ Aún así somos conscientes de que el auténtico motor del desarrollo lingüístico es el contacto con la lengua misma y de que se trata de estrategias que pueden contribuir a la capacidad comunicativa puesta a prueba a través de ejercicios que simulen la comunicación, pero que sólo se mostrará de forma real en situaciones comunicativas o actos de habla reales.

${ }^{22}$ C. Terzan-Kopecky, «Zu den Verbalkategorien Aspekt und Tempus». En: Deutsch als Fremd-sprache 1, Leipzig, 1998, pp. 17-23. 
sintética (8) a la analítica (9), debido principalmente a la frecuencia con la que se producen a nivel oral, en el transcurso de una conversación por ejemplo, referencias temporales de todo tipo: oraciones subordinadas temporales, semantemas temporales, o la contextualización pragmática sin referencias temporales ${ }^{23}$. Otro ejemplo podría ser la pogresiva sustitución de la forma sintética Präteritum por la forma analítica haben / sein + Perfekt, o dicho de otro modo, la relación existente entre el uso del sistema temporal a nivel escrito / hablado, o la progresiva tendencia del uso indistinto de Perfekt / Präteritum en la lengua hablada o escrita debido más a una cuestión de estilo que a la diferenciación semántica o a razones morfosintácticas ${ }^{24}$. Con estos ejemplos queremos ilustrar que realidades, como la anteriormente citada, pertenecientes al conocimiento sobre el uso lingüístico, pueden ser tematizadas. La cuestión es cómo y en qué momento. Lo que sí consideramos es que benefician al estudiante de lenguas extranjeras en el perfeccionamiento de su competencia comunicativa, pues le sitúan muy cerca de la realidad en la que se verá inmerso.

La reflexión interactiva nos conduce a un último punto controvertido: la pertinencia o no del análisis contrastivo, o confrontativo con la lengua materna. La gramática contrastiva puede afrontar perfectamente aspectos funcionales de la descripción lingüística. En la medida en que el análisis contrastivo, encaminado principalmente a la profilaxis de errores $^{25}$, persiga objetivos comunicativos, puede contribuir positivamente a disipar dudas sobre usos divergentes en las dos lenguas, la materna y la lengua objeto de estudio, o tres, si podemos recurrir a una lengua extranjera aprendida con anterioridad a la lengua alemana, por ejemplo.

El análisis contrastivo se manifiesta como verdaderamente efectivo al incidir en aspectos problemáticos que se manifiestan en el proceso de transmisión del INPUT a nivel gramatical. Sirva de ejemplo la diferencia entre el sistema temporal español, dotado de 17 tiempos gramaticales frente a los 6 del sistema alemán ${ }^{26}$. Como es lógico, es muy difícil establecer una correspondencia semántica entre ambos sistemas, especialmente en el ámbito del pasado, pues al margen del uso del Plusquamperfekt, coincidentes en ambos, al Präteritum alemán corresponden, por ejemplo, los tiempos españoles pretérito indefinido $e$ imperfecto. La reflexión sobre las divergencias existentes contribuirá positivamente al uso adecuado de formas del tipo sabía / supe o conocía / conocióo ${ }^{27}$ en lengua alemana. Es cierto, que el estudiante, sólo en contadas ocasiones es consciente del funcionamiento del sistema lingüístico materno, por lo que, en opinión de algunos lingüistas, en estos casos el

${ }^{23}$ Vid. C. Terzan-Kopecky, op. cit., p.17.

${ }^{24}$ M. Hennig, «Tempus - gesprochene und geschriebene Welt?». En: Deutsch als Fremdsprache, 4, Leipzig, 1998, pp. 227-232.

25 Vid., por ejemplo. Mulo, op. cit., p. 158

${ }^{26}$ K., Forster, «Lernschwierigkeiten und Fehlerursachen im Kontrast Spanisch-Deutsch: Morphologie». En: Deutsch als Fremdsprache, 4, 1994, pp. 209-215.

${ }^{27}$ K. Forster, op. cit, p. 213. 
análisis confrontativo resulta inadecuado y poco menos que contraproducente. Pero quizá sea la única opción para que el docente pueda establecer las diferencias y favorecer el uso correcto en la lengua extranjera, evitando de esta forma las frecuentes traducciones literales que producen una transmisión errónea de información y que dificultan seriamente la comunicación, y por lo tanto, la competencia comunicativa del discente. Así, el problema que plantea la diferenciación entre pretérito indefinido e imperfecto, (fundamentada más en divergencias aspectuales, acción perfectiva o durativa, que en una ordenación de la dimensión "tiempo"), y el uso homólogo en lengua alemana habrá de ser solucionado con la aplicación de verbos diferentes:

PRETÉRITO INDEFINIDO

sabía

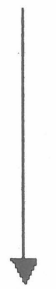

ich wusste

\section{PRETÉRTTO IMPERFECTO}

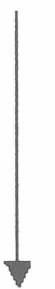

\section{PRÄTERITUM}

El análisis contrastivo, como hemos mencionado con anterioridad puede evitar así las traducciones del tipo se conocieron por sie kannten sich y contribuir a clarificar las correspondencias:

$$
\begin{array}{ll}
\text { se conocían } & \text { sie kannten sich } \\
\text { se conocieron } & \text { sie lernten sich kennen }
\end{array}
$$

Problemático resulta, igualmente, el uso del pretérito perfecto español frente al la forma analítica Perfekt y la sintética Präteritum, comentadas con anterioridad o la correcta aplicación de los verbos auxiliares haben / sein que da lugar a numerosas formas erróneas como las citadas en (10) o a la necesidad de clarificar usos como en (11):

(10) *er hat gelaufen / er hat eingeschlafen / er hat angekommen

(11) er ist mit dem Auto gefahren / er hat das Auto gefahren

Estamos convencidos de que, sin dar la espalda a los objetivos comunicativos y en casos de absoluta necesidad, este tipo de estrategia resulta muy adecuada para evitar posibles interferencias entre ambas lenguas o incluso una tercera, como la lengua inglesa, por 
ejemplo, de dominio general entre estudiantes hispanohablantes. No 'debe, en cambio, constituir una estrategia de uso generalizado, pues cuando no existen divergencias importantes entre lengua materna y lengua extranjera difícilmente se producen interferencias.

La reflexión gramatical a través de la acción interactiva debe venir apoyada por un ejercicio, siempre contextualizado, en el que el estudiante pueda poner a prueba las conclusiones a las que ha llegado: debe tener la oportunidad de reconocer las dificultades comentadas, de supervisar su producción lingüística de forma crítica. Este ejercicio no tiene sentido si no ha habido una reflexión y sensibilización anterior, pues de no ser así nos llevaría al infructuoso ejercicio de drills automatizados o a los no menos engañosos chunk learnings. En definitiva, a modelos de aprendizaje que, en ocasiones, se manifiestan en la consecución de objetivos de orientación comunicativa como ineficaces o poco apropiados, más por cuestiones metodológicas que por la naturaleza del ejercicio.

Podemos concluir afirmando que la programación de contenidos gramaticales, en nuestro caso la didactización de los contenidos en torno al tiempo verbal, debe efectuarse siempre en función de unos objetivos comunicativos. La unidad forma / función debe manifestarse también a nivel metodológico, aplicando estrategias encaminadas siempre al desarrollo de la capacidad comunicativa del estudiante, en este caso, de lenguas extranjeras. La función nos debe llevar a la forma y ésta debe llegar al discente fundamentada en una metodología basada en la interacción y el aprendizaje consciente. Ejercicios emancipatorios resultan de gran interés en el desarrollo de la competencia comunicativa y por lo tanto, indirectamente en la aplicación de reglas gramaticales. Estamos convencidos de que este tipo de metodología contribuye a que el discente se acerque de forma interesada a las normas del sistema y aprenda a valorarlas en su justa medida, como parte esencial del proceso comunicativo.

\section{BIBLIOGRAFIA}

Arbeitsbruppe Fremdsprachenerwerb Bielefeld (1995), «Fremdsprachenerwerbsspezifische Forschung. Aber wie? Theoretische und methodologische Überlegungen (I)». En: Deutsch als Fremdsprache. 3, 1996, Leipzig, pp. 144-155.

Arbeitsbruppe Fremdsprachenerwerb Bielefeld (1995), «Fremdsprachen erwerbsspezifische Forschung. Aber wie? Theoretische und methodologische Überlegungen (II)». En: Deutsch als Fremdsprache, 4, 1996, Leipzig, pp. 200-210.

Blühdorn, H., «Deutsch als Fremdsprache und die Grammatik des Deutschen. über die Fruchtbarkeit des fremden Blicks». En: Deutsch als Fremdsprache, 4, 2000, pp. 221-226.

Bogner, A., «Fremdsprachenerwerbsforschung zwischen Kultur- und Naturwissenschaft». En: Jahrbuch Deutsch als Fremdsprache, 25, 1999, pp. 29-49.

Deutrich, K. / G. Schank, «Situationsspezifischer Einsatz sprachlicher Mittel. Anregungen für den Deutschunterricht». En: Der Deutschunterricht 6, 1973, pp. 100-120.

Diehl, E., «Vom Deutschunterricht zum Deutscherwerb? Das Genfer DiGS-Projekt». En: Deutsch als Fremdsprache, 3 1998, pp. 162-168. 
Eichler, W., «Strukturelle Sprachbetrachtung im Deutschunterricht». En: Der Deutschunterricht, 4, 1970, pp. 99-113.

Fiehler, R. «Bewertungen und Normen als Problem bei der Förderung von Gesprächsfähigkeiten». En: Der Deutschunterricht, 1, 1998, pp.53-64.

Forster, K. «Lernschwierigkeiten und Fehlerursachen im Kontrast Spanisch-Deutsch: Morphologie». En: Deutsch als Fremdsprache, 4, 1994, Herder-Institut, Leipzig, pp. 209215.

Götze, L., «Grammatik und Kommunikation - ein Widerspruch? En: Deutsch als Fremdsprache, 3,1991, Herder-Institut, Leipzig, , pp. 161-163.

_, «Grammatikmodelle und ihre Didaktisierung in Deutsch als Fremdsprache». En: Deutsch als Fremdsprache 3, 1996, pp. 136-143.

Grünwaldt, H., «Zur Didaktik und Methodik mündlicher Kommunikationsübungen». En: Der Deutschunterricht, 1, 1998, pp.65-73.

Harnisch, H. / Georg Michel, «Fremdsprachenunterricht und funktional-kommunikative Sprachbeschreibung». En: Deutsch als Fremdsprache 1, Leipzig, 1985, pp. 8-14.

Helbig, G., «Kommunikativer Grammatikunterricht -Ziele, Möglichkeiten und Grenzen». En: Deutsch als Fremdsprache, 1, Leipzig, 1986, pp. 14-20.

Hennig, M., «Tempus - gesprochene und geschriebene Welt?». En: Deutsch als Fremdsprache, 4, Leipzig, 1998, pp. 227-232.

Homberger, D., «Information-Grundbegriff im Deutschunterricht». En: Der Deutschunterricht, 6, 1971, pp. 5-19.

Kasjan, A., «Deutschunterricht unter erschwerten Bedingungen: Grammatik als Risiko und Chance». En: Deutsch als Fremdsprache, 4, Leipzig, 1998, pp. 168-171.

Kolsanskij, G.V., «Komuunikationslinguistische Probleme und Fremdsprachenunterricht». En: Deutsch als Fremdsprache, 2, Leipzig, 1985, pp. 73-77.

Latzel, S., «Die Vergangenheitstempora als Lehr- und Lerngegenstand im Fach “Deutsch als Fremdsprache”. En: Zielsprache Deutsch, 4, München, 1974, pp. 151-166.

Mulo, B., (1996): «Aspekte einer kommunikativ-funktionalen Grammatik aus der Fremdperspektive». En: Deutsch als Fremdsprache”, 3, pp. 156-159.

Nespital, H. «Verbbedeutung und Aspekt aus sprachvergleichender Sicht». En: Sprachwissenschaft, T.8,4, 1983, pp. 357-384.

Neuner, G., «Deutsch als Fremdsprache nach Englisch. Überlegungen zur Didaktik und Methodik und zur Lehrmaterialentwicklung für die "Drittsprache Deutsch"». En: Deutsch als Fremdsprache. 4, Leipzig, 1996, pp. 211-217.

Reinecke, W., «Zum Verhältnis von grammatischer Paradigmatik und Syntagmatik bei der Aneignung von Fremdsprachen». En: Deutsch als Fremdsprache 5, Leipzig, 1985, pp. 257-260. 
Rusch, P., «Schritte zum Ausbau einer Lernergrammatik». 'En: Deutsch als Fremdsprache 4, Leipzig, 1998, pp. 233-238.

Sendel`s E.I., «Pragmatik, Semantik und Konnotation». En: Deutsch als Fremdsprache, 2, Leipzig, 1985, pp. 84-88.

Sitta. H. / Tymister, H., Linguistik und Unterricht, Tübingen, Max Niemeyer Verlag, 1978.

Sternemann, R., «Konfrontative Linguistik und Deutsch als Fremdsprache. Eine kritische Musterung». En: Deutsch als Fremdsprache, 2, Leipzig, 1983, pp. $65-70$.

Terzan-Kopecky, C., «Zu den Verbalkategorien Aspekt und Tempus». En: Deutsch als Fremdsprache 1, Leipzig, 1998, pp. 17-23.

Trost, K. «Verbalaspekt». En: Sprachwissenschaft, T. 2, 1, 1977, pp. 1-26.

Thurmair, M., «Nicht ohne meine Grammatik!». En: Jahrbuch Deutsch als Fremdsprache, 23, 1997, pp. 25-45.

Vennemann, T., «Tempora und Zeitrelation im Standarddeutschen». En: Sprachwissenschaft, T. 12, 2, pp. 234-249.

Wolff, G., «Sprechakte im sozialen Kontext». En: Der Deutschunterricht, 6, 1973, pp. 19-49.

Wragge, I., «Kommunikationsfähigkeit als Aufgabe des Deutschunterrichts. Ein Beitrag zur Didaktik des Sprachtrainings». En: Der Deutschunterricht, 3, 1974, pp. 82-98. 